Document downloaded from:

http://hdl.handle.net/10251/155403

This paper must be cited as:

Marco, FJ.; Martínez Uso, MJ.; López, JA. (2018). A 3D-Study of the residual vector field. Proceedings of the International Astronomical Union. 12(S330):235-236. https://doi.org/10.1017/S1743921317006536

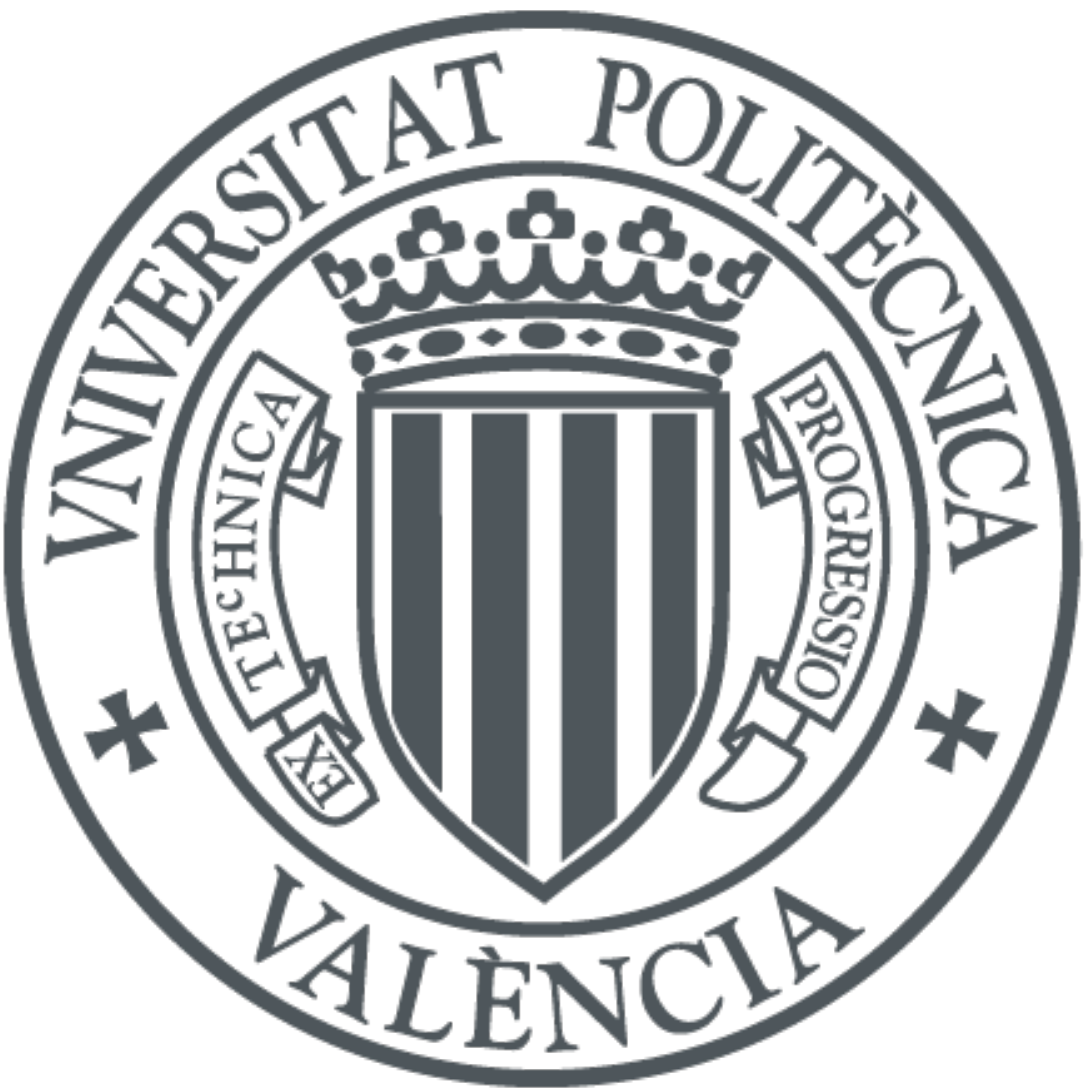

The final publication is available at

https://doi.org/10.1017/S1743921317006536

Copyright Cambridge University Press

Additional Information 


\title{
A 3D-Study of the residual vector field
}

\author{
Francisco J. Marco ${ }^{1}$, María J. Martínez ${ }^{2}$ and Jose A. López ${ }^{1}$ \\ ${ }^{1}$ Universidad Jaume I. Dept. Matemáticas, IMAC. \\ Avda. Vicent Sos Baynat s/n, 12071, Castellón, Spain \\ email: marco@mat.uji.es, lopez@mat.uji.es \\ ${ }^{2}$ Universidad Politécnica de Valencia. Dept. Matemática Aplicada. IUMPA. \\ Camino de Vera s/n. 46022 Valencia. Spain \\ email: mjmartin@mat.upv.es
}

\begin{abstract}
One of the important challenges that Gaia imposes on the Astrometric Catalogs, is a careful study in everything affected by parallax. A particularly important case is the necessary linkage Gaia - HCRF - ICRF2, which require methods of analysis that are accurate enough so that the provided results are at the same precision level as the work data.
\end{abstract}

Keywords. astronomical data bases: miscellaneous, catalogs, reference systems

\section{Introduction}

We will consider the usual discrete points for which we know the Right Ascension, the Declination, and the trigonometric parallax $p$. We denote $r=1 / p$. We will use Hipparcos2 and UCAC4 catalogs. Regarding the limitations in some measurements in both catalogues, this paper represents an initial approximation to a further work that will be done using the new Gaia catalogue. We consider the residuals $\Delta \alpha^{*}, \Delta \delta$ together with their corresponding $r$ values. These residuals can be seen as unidimensional random variables (r.v.) A regression on $r$ shows that there exist dependencies $\Delta \alpha^{*}(r), \Delta \delta(r)$. For a r.v., we calculate the regression as:

$$
E[X \mid r]=\frac{\int x f(x, r) d x}{\int f(x) d x}
$$

where each $f$ represents different density functions, depending on the context. Our final aim is to consider the vector field $\left[\Delta \alpha^{*}(r), \Delta \delta(r)\right]$ for the $\mathrm{N}=82446$ selected stars- Due to low density of the 3D-discrete, we have decided to work in "slices" of 200 pcs. Here, we focus on a particular slice centered at 400 pcs.

\section{Methods}

Adjusting a three-dimensional spherical function

$$
\widehat{m}(\alpha, \delta, r)=\sum_{i=1}^{n} \varpi_{i} z_{i}
$$

where $z_{i}=\Delta \alpha_{i}^{*}\left(r_{i}\right)$ or $\Delta \delta_{i}\left(r_{i}\right)$ and $\varpi_{i}$ are the weights for the Nadaraya-Watson method in the three-dimensional case. The low three-dimensional density recommends the use of a relatively high bandwidth in $r$ Vector spherical harmonics (VSH) development

Denoting by $Y_{n m}, n \geqslant 0,|m| \leqslant n$, the usual spherical surface harmonics, the VSH are:

$$
\vec{R}_{n m}=\vec{r} Y_{n m}, \vec{S}_{n m}=\overrightarrow{\nabla Y}_{n m}, \vec{T}_{n m}=-\vec{r} \times \overrightarrow{\nabla Y}_{n m}
$$

They are an orthogonal and complete base in the Hilbert space of the vector fields $L^{2}\left(S^{2}\right)$. 

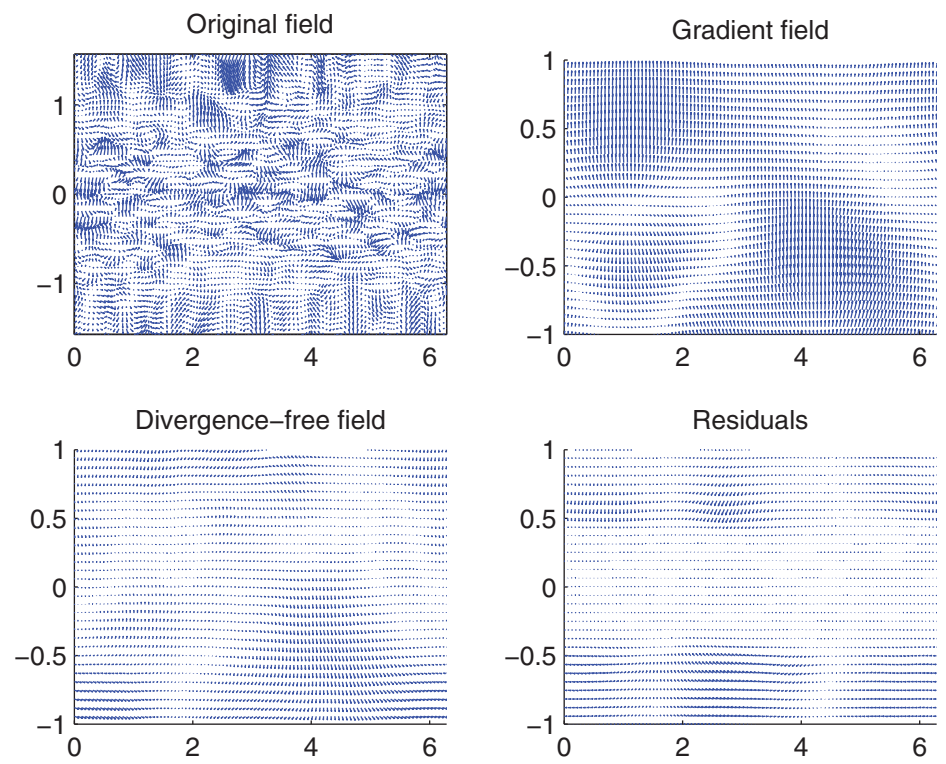

Figure 1. Original field, gradient field, divergence-free field and residual field.

Table 1. Div-free and curl-free components for the residual vector fields around $r=400$ pcs

\begin{tabular}{|l|l|c|c|c|c|c|c|}
\hline$r$ (pcs) & & $s_{1,0}$ & $s_{1,1}$ & $s_{1,-1}$ & $t_{1,0}$ & $t_{1,1}$ & $t_{1,-1}$ \\
\hline 400 & $\begin{array}{l}\text { Curl-free } \\
\text { Div-free }\end{array}$ & 8.72 & 7.90 & 1.50 & --04 & -- & -- \\
& Difo & -4.20 & -0.09 & 3.38 & -0.08 & 3.61 \\
\hline
\end{tabular}

Helmholz Decomposition We know that we can obtain a divergence-free vector from a noise vector. This is a potential source of errors and that is why we propose, as a method of analysis of coefficients and errors, to perform previously a Helmholtz decompositions for each especially difficult vector fields.

Let us consider a field on the sphere $\vec{X}$, Helmholtz theorem provides a (unique) decomposition: $\vec{X}=\overrightarrow{\nabla \phi}+\vec{\nabla} \times \vec{u}+\vec{\varepsilon}$ where the gradient of the potential, $\overrightarrow{\nabla \phi}$, is curl-free, the curl $\vec{\nabla} \times \vec{u}$ is divergence-free and the residual field $\vec{\varepsilon}$ verifies both properties. From this point, we recompute the VSH coefficients again.

\section{Results}

Certain 3D regions should be studied in more detail, due to the variation in the values of certain coefficients. We have taken the neighbourhood of 400 pcs. Here, the distances between residuals in RA and Dec are relatively large. For RA, the residuals increase near a local maximum and decrease near a local minimum for Dec. This must reflect in the vector field. In Table 1 we show the results for $r=400 p c s$. The complete, curl-free, divergence free and remnant $(\vec{\varepsilon})$ vector fields may be seen in Figure 1.

We should point out that UCAC4 is not independent of the Hipparcos catalogue, as Tycho-2 stars are used as reference for UCAC4. This makes more difficult the interpretations of the residuals, which will be discussed in a further paper.

\section{Acknowledgements}

This work was supported by a grant UJI-B2016-18. 